\title{
COMPARING FUNCTIONAL AND CELLULAR LAYOUTS: SIMULATION MODELS
}

\author{
Jerbi, A. ; Chtourou, H. ${ }^{* * *}$ \& Maalej, A. Y. \\ * Laboratoire des Systèmes Electro-Mécaniques, Ecole Nationale d'Ingénieurs de Sfax, \\ BP W 3038, Sfax, Tunisia \\ *** Département de technologie, Institut Préparatoire aux études d’Ingénieurs de Sfax, \\ Rte Menzel Chaker Km 0.5, Sfax 3018, Tunisia \\ E-Mail: jerbi_a@yahoo.fr, hedi.chtourou@ipeis.rnu.tn, aref.maalej@enis.rnu.tn
}

\begin{abstract}
The cellular layout has been compared to the traditional functional layout in numerous studies. Most of them are based on simulation modelling. Nevertheless, they generally suffer from the lack of detail in describing their simulation models. The exclusion or the lack of information of key parameters could be serious limitations to any simulation study. The main purpose of this work is the development of comprehensive FL and CL simulation models and their use for illustrating the dependence of the layout performance comparison on the settings of factors either overlooked or kept constant by several simulation studies. Simulation results clearly establish the influence of part type routings, cellular queuing strategy and job overlapping on the ranking of the two layouts.

(Received in June 2009, accepted in August 2009. This paper was with the authors 1 month for 1 revision.)
\end{abstract}

Key Words: Manufacturing Systems, Cellular Layout, Functional Layout, Simulation

\section{INTRODUCTION}

Since its apparition and the spreading out of the group technology concept, the cellular layout (CL) has been compared to the traditional functional layout (FL). Analytical models and empirical research have often been used for the two manufacturing system (MS) layout patterns. However, the major part of the literature devoted to FL-CL comparison is based on simulation modelling.

Results reported by comparative simulation studies vary widely. Indeed, some researches reported that the FL is always superior to the CL with regard to all used performance measures [1-3]. In contrast, further researches found the CL superior to the FL in all operating conditions [4], [5]. Some other simulation studies established that each layout could outperform the other in some particular experimental conditions [6-10]. This divergence in the conclusions is referred as the "cellular manufacturing paradox" [11]. [12] studied this paradox as it emerged from a number of comparative simulation studies. It identified a number of objectivity flaws responsible for the conflicting conclusions. Prior to this study, [11] and [13] reviewed and analyzed a number of FL-CL comparative studies. Their works simply reported the major findings of some published studies without any critical objectivity assessment.

In fact, it appeared from [12] results that some parameter settings, such as under sizing transfer times or excluding job overlapping in CL, biases the comparison in favour of the FL. On the other hand, some other settings favour the CL. Among these settings, machine duplication and very high transfer times can be mentioned. Besides, the same study pointed out the fact that many potentially influential factors were considered constant throughout some of the simulation investigations. In addition, according to the same study, some of the 
reviewed studies failed to describe their simulation models with a sufficient amount of detail. The lack of data concerning important factors or excluding these factors from the simulation investigation could be serious limitations to any simulation study.

The main purpose of this research is to develop comprehensive FL and CL simulation models using the commercial simulation software Arena 7.0. These models are then used to illustrate the dependence of the layout performance comparison on the settings of factors either overlooked or kept constant by several studies. The remainder of this paper is organized as follows: the next section presents taxonomy of foremost used performance measures in the main published simulation studies concerned with FL-CL comparison. This section also discusses the main used factors. The third section presents some general simulation features, needed for modelling both layouts. Then, sections 4 and 5 respectively detail the particularities of the developed FL and the CL simulation models. Next, section 6 presents a simulation study illustrating the dependence of the comparison results on several parameters, often overlooked in the literature. The final section encloses the general conclusion and the future work prospects.

\section{LITERATURE REVIEW}

\subsection{Performance measures}

The Work In Process (WIP) is one of the most popular performance measures used in the FLCL comparative studies [1-4], [6-8], [10]. This measure characterizes the fluidity of the material flow in the system. It has been mainly measured by the number of parts in the system or by summing up the average setup and process times of all parts either being processed or waiting in the different MS queues. The Mean Flow Time (MFT) constitutes the other most popular measure used in FL-CL comparative studies [1], [3], [4], [6-10]. The MFT is the average time that every job remains in the system in order to be manufactured. It also characterizes the system flow fluidity.

In addition, researchers used Mean Tardiness $(M T)$ and Mean Earliness $(M E)$ as due date related performance measures [3], [8]. The first is taken as the average, over all tardy jobs, of the difference between actual delivery date and the promised due date. The second is similarly obtained for all early jobs. [3] also uses the percentage of tardy jobs and the percentage of early jobs.

FL-CL comparative studies consider several other performance measures. The system throughput is usually considered as a productivity measure. It is the average number of parts exiting the system by time unit [2], [9]. In addition, some studies use the average machine utilization rate [2], [7], [8], the operator utilization rate [2], the average set up time to process time ratio [10] or the mean "queue" waiting time as performance indicators [5]. Maximising the first two measures ensure a high degree of resource exploitation whereas minimising the third or the fourth measure enhances the efficiency of the MS piloting.

\subsection{Main experimental factors}

The studied MSs are characterized by a number of machines ranging from 12 to 30 . These are either arranged into departments or else into manufacturing cells. Following the FL structure, the shop is composed of $d$ departments $D_{i}(i=1, \ldots, d)$ each of them includes $M_{n}$ functionally equivalent machines. In contrast, the CL, based on the group technology, is composed of $c$ independent manufacturing cells $C_{j}(j=1, \ldots, c)$. Each one of these cells is a cluster of $M_{f}$ different machines dedicated to a number of similar part types. In the studied MSs, the 
number of departments ranges between 4 and 15 whereas the number of cells ranges between 3 and 5.

The Degree of Decomposability ( $D D$ ) of the part machine matrix translates the feasibility of the decomposition of the MS into independent cells. Indeed, the more the product/machine matrix is diagonal the more the decomposability is feasible. The majority of the comparison studies considered very high degrees of decomposability leading to completely independent cells.

Products are identified by two indicators, which are the type $(t)$ and the family $(f)$. In fact, different product types are regrouped into families according to the similarity of their manufacturing process. The studied systems were designed for a demand pattern comprising 3 to 80 product types belonging to a number of families varying from 3 to 10 . Each product type requires a number of manufacturing operations (mopt) ranging between 2 and 25.

Jobs arrive to the MS following the InterArrival Time (IAT) that represent essentially the demand rate of the MS. A large part of authors generated this time by common probabilistic distributions. Others used a constant interarrival time.

Once jobs arrive into a department or a cell they are made waiting in a queue until the required machine becomes available. These jobs are then sequenced in order to establish the priority of jobs and the order in which they will be processed. This order is specified by a scheduling rule or a priority rule. Common rules include "First Come First Served" (FCFS), "Shortest Processing Time" (SPT), "Earliest Due Date” (EDD) and "Repetitive Lots" (RL).

As for the IAT, several studies modelled the Processing Time (PT) and Setup Time (ST) by different probabilistic laws. Others formulated $S T$ as a fraction of $P T$. In addition, if a machine is set up for a specific job type and then it should be set for another type of the same family, the nominal setup time for the second job should be reduced by the reduction time factor $(\delta)$.

Products are generally manufactured and transported in batches $(B S)$ which size ranges between 5 and 250 parts in order to reduce the number of machines' setup and transport between workstations. Nevertheless, different MSs use the operations overlapping as a transfer mode that allows simultaneous execution of different operations on the same batch. Therefore, they exploit the proximity of cell machines in the cellular layout and transferred products by part.

Neither operator's number nor skill was considered in the quasi totality of FL-CL comparative studies. Indeed, the mainstream of literature assumes that an operator is affected to every machine.

\section{GENERAL SIMULATION FEATURES}

Both FL and CL layouts are modelled for simulation using the commercial simulation software Arena 7.0 [14-16]. This tool integrates all the functions needed for simulation including animation, analysis of input and output data. Every MS model consists of the four main components described in the following subsections.

\subsection{Manufacturing orders launching and attribute assignation}

Manufacturing orders (MOs), also called jobs, are launched by "Create" modules that permit to define jobs interarrival times following an appropriate probabilistic rule as well as their batch size. Jobs of each part type are launched by a dedicated "Create" module. As soon as jobs are initiated, several characteristics are attributed to them using the "Assign" module. 
These characteristics are mainly job's type and family. Routings, process and setup times on each machine type belonging to their routings are also assigned to jobs by the same module.

\subsection{Part transfer}

Parts are transferred, either individually or in batch, between physical locations in which they should undergo the required successive manufacturing operations. These locations, modelled by the "Station" modules, are either machines in CL or departments in FL. Transfer is modelled by "Route" modules permitting to prescribe destinations as well as transfer times. These modules permit to use one of the previously defined jobs attributes, namely its "Sequence", in order to prescribe the next destination. The Job "Sequence" corresponds to its routing expressed as a list of stations. These stations are associated either to departments in the FL or to the cell machines in the CL.

\subsection{Part manufacturing}

A machine is modelled by a "Process" module, associated to a "Station" module determining the physical location of the machine and a "Resource" module representing the capacity and the availability of the machine itself. In fact, the "Process" module seizes the associated resource for the required period of time and then releases it, so it becomes again available for manufacturing another job. The period of time during which the machine resource is busy corresponds to the $P T$ of the part being processed as well as the required machine $S T$ if the machine was set for a different part type. The $S T$, when applicable, is weighed by the setup reduction factor $\delta$ whenever the part type belongs to the family of the last processed one.

\subsection{Material flow}

Two manufacturing strategies could be followed for the parts flow in the shops. In the first, job overlapping is allowed. This means that parts of the same batch could be processed simultaneously on the different machines of a department or a cell, depending on the used layout. In the second, overlapping is not permitted. Hence, all parts of the same batch are processed on the same machine of the cell or department before being transferred collectively to the next machine or department prescribed by their routing. In all cases, batches should be split by "Separate" modules before accessing "Process" modules. Batch reconstitution for transfer is performed using "Batch" modules.

\subsection{Statistics collection}

Before leaving the MS, every job should go through an “Assign” module for computing and updating the parameters defined as performance measures. The acquired data is then stored in an Excel file using a "Readwrite" module for eventual treatment and analysis.

\section{FL MODEL}

The functional layout model is mainly composed of three parts: "MO launching", "Machine departments" and "System exit" (see Fig. 1). 


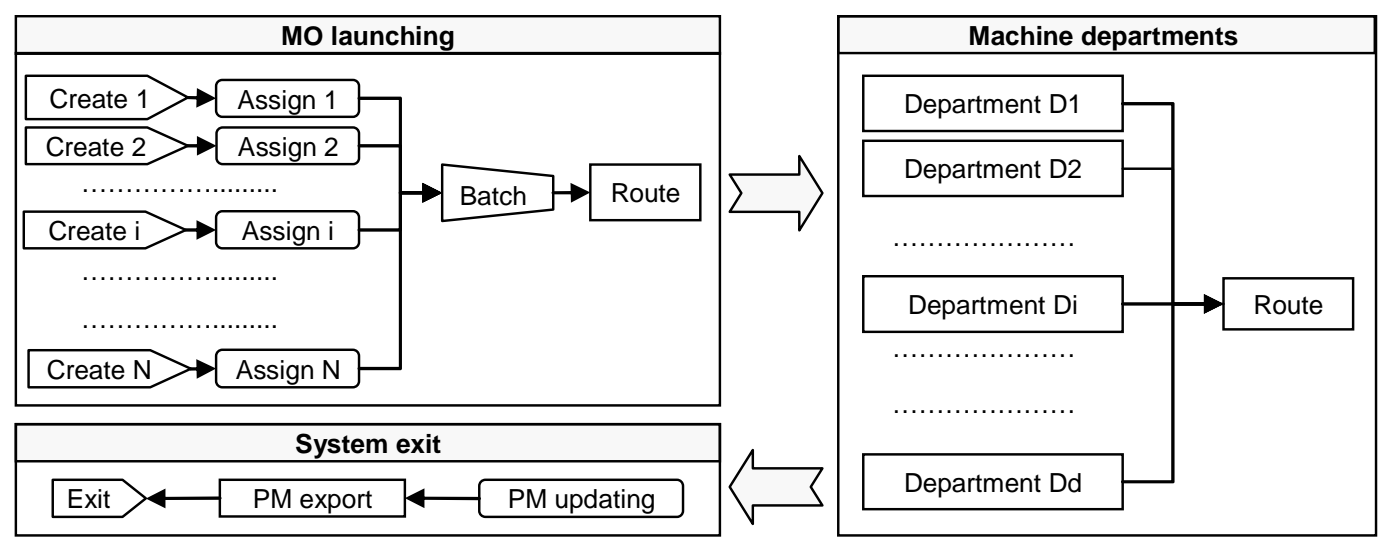

Figure 1: Overview of the FL model.

MOs of each part type are launched by dedicated "Create" and "Assign" modules. The generated parts are then grouped into batches before being routed to the corresponding department to their first manufacturing step. Once a job enters a department, it waits in a queue modelled by the "Hold" module and governed by a rule that could be FCFS, SPT or any other priority rule specified by the user. In this study, the RL priority rule is used for both FL and CL models. This rule aims at minimising the setup times on the machines by prioritising jobs of the same type than the job just processed on one of the department machines. If no matched job is found, jobs of the same family are selected. If still no matched job is found, the queue is scanned for a job type different from those being processed on the others department machines. Finally, the selection procedure is switched to the FCFS priority rule if the prior searches fail to produce a candidate.

The "Hold" module releases the prioritized job of the waiting queue when, at least, one machine is available. The released job is then transferred to the "Machine selection" submodel. This sub-model is responsible for selecting one among the available department machines. The logic of this sub-model is coherent with the waiting queue priority rule. Hence, it routes the released job to the appropriate machine according to the RL.

In addition, when job overlapping is permitted, parts batches are split just before accessing the department queue. Hence, parts become independent and could be dispatched to several machines of the same department to be processed simultaneously (see Fig. 2). On the other hand, if overlapping is not adopted, every parts batch is split only when it reaches the machine assigned to its processing (see Fig. 3). In both cases, batches are reconstituted after processing and before the transfer to next manufacturing step. The combination of the overlapping strategy, the machine selection process and the waiting queue priority rule define the shop scheduling policy.

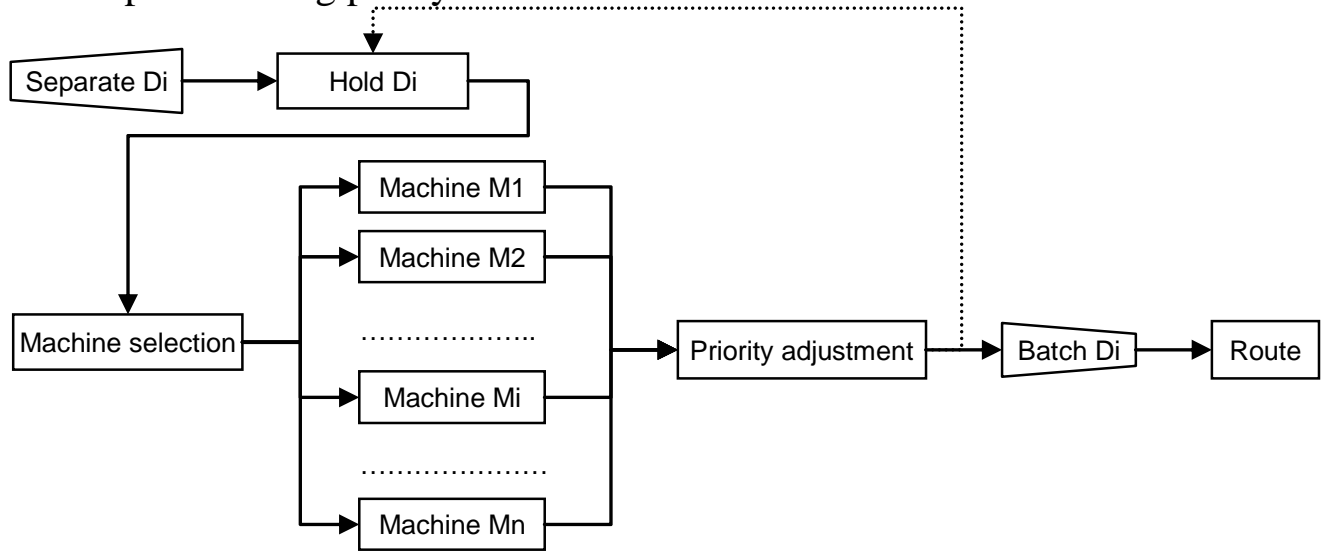

Figure 2: Overview of an FL department model with overlapping. 


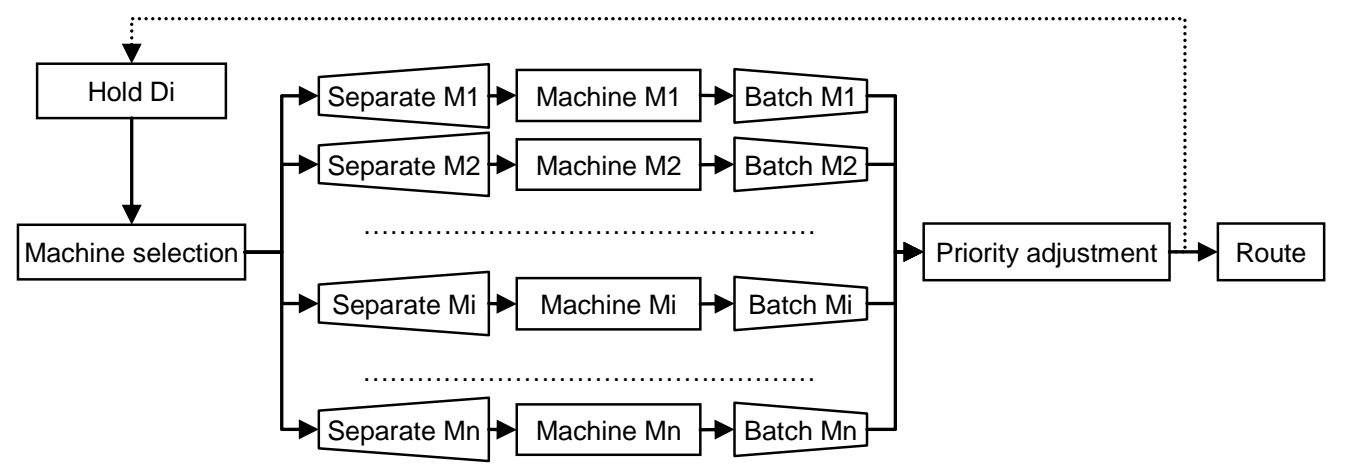

Figure 3: Overview of an FL department model without overlapping.

\section{CL MODEL}

The CL model is composed of $c$ sub-models corresponding to the $c$ MS cells. Each sub-model is composed of three parts: "MO launching”, “Machine cells" and “Cell exit” (see Fig. 4).
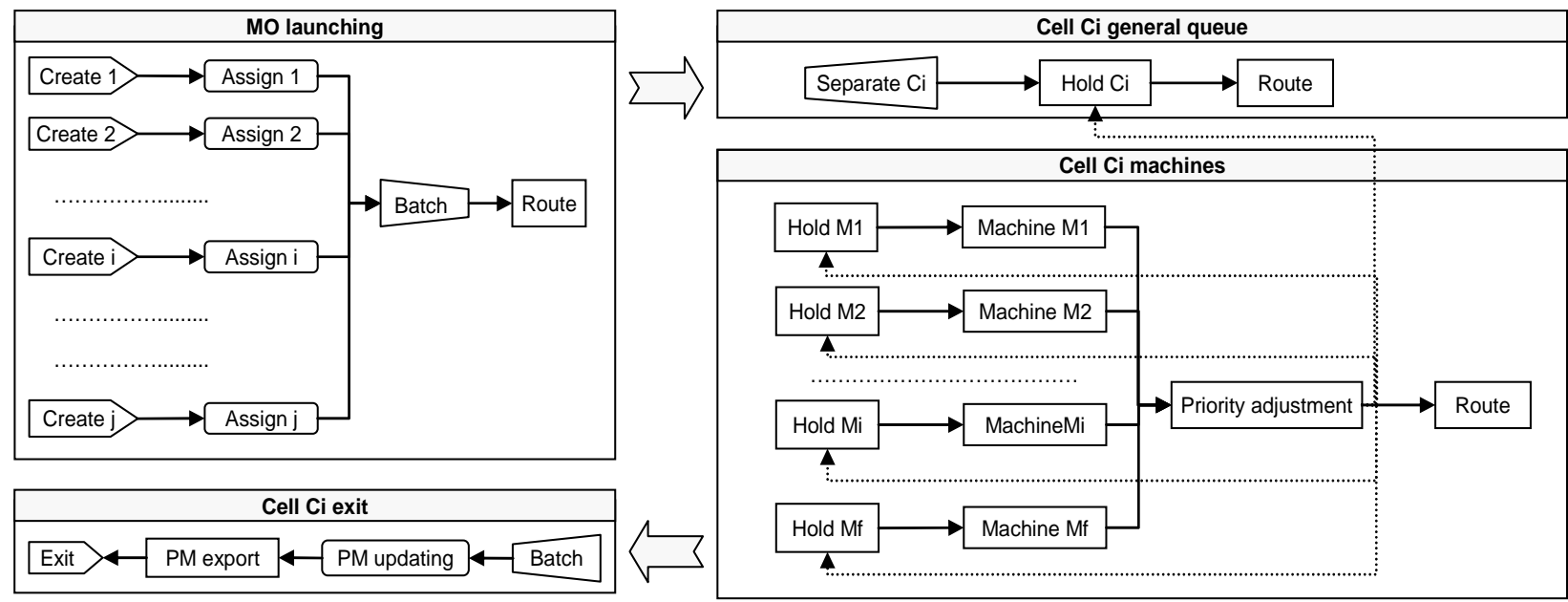

Figure 4: Overview of a cell $\mathrm{C}_{\mathrm{i}}$ model with overlapping.

MOs of each part type belonging to the cell part type family are launched by dedicated "Create" and "Assign" modules. The generated parts are then grouped in batches before being routed to the machine corresponding to their first manufacturing step. Each machine has its own waiting queue governed by a specified priority rule. An additional general cell queue could also be implemented. Such a queue holds parts until their first routing step machine becomes available before being inserted into the cell. Both queues are governed by the same RL priority rule. Once a machine becomes idle, a double stage strategy is adopted for the job selection. Jobs are first selected from the machine queue. Once this queue is void, jobs are selected from the general cell queue.

As for FL, process overlapping could be allowed. In this case, batches are dissociated since they leave the cell general queue. Hence, every part follows its manufacturing routing without waiting for the other batch parts. Batches are finally reconstituted just before the cell exit (see Fig. 4). On the other hand, if overlapping is not adopted, every batch is split only when it reaches the machine assigned to its processing. Batches are then reconstituted after processing and before the transfer to next manufacturing step (see Fig. 5). 


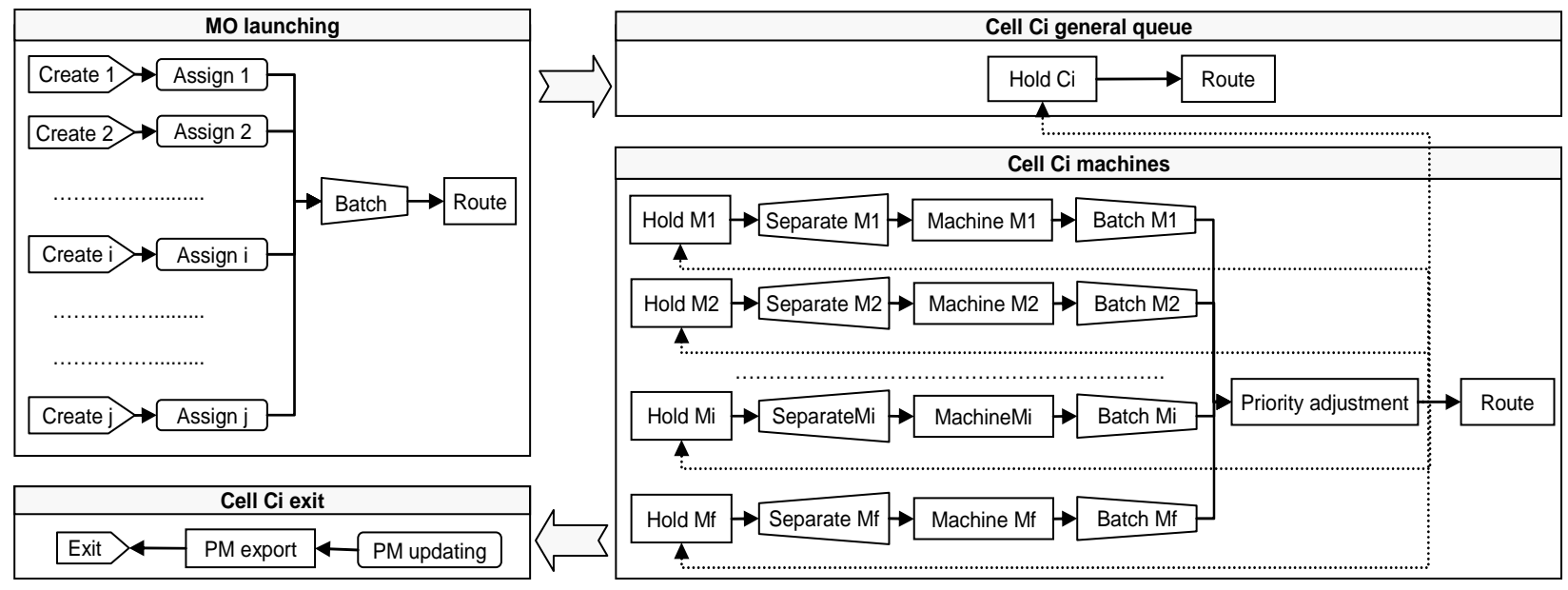

Figure 5: Overview of a cell $C_{i}$ model without overlapping.

\section{SIMULATION STUDY}

\subsection{Scope}

The developed simulation models are used to illustrate the dependence of the FL-CL comparison results on several parameters often overlooked in the literature. These factors are part type routings, cell queuing strategy and job overlapping strategy. Hence, three simulation experiments are carried out. Table I summarises characteristics of both layouts.

Table I: MS characteristics.

\begin{tabular}{|l|l|}
\hline Characteristic & Value \\
\hline No. of parts / families & 30 Parts / 5 part families \\
\hline Family size & 6 parts/family \\
\hline No. of machines & 30 \\
\hline No. of cells & 5 \\
\hline No. of departments & 8 \\
\hline Cell size & $4 \sim 8$ machines/cell \\
\hline Labour & 30 operators \\
\hline Functional department size & $3 \sim 4$ machines/department \\
\hline No. of operations/part & $2 \sim 6$ \\
\hline Batch size & 50 parts \\
\hline Job interarrival times & Exponential distribution with mean $=630$ min \\
\hline Process times & Normally distributed with mean $=20$ min and variance $=2.5$ min \\
\hline Setup times & Normally distributed with mean $=100$ min and variance $=12.5$ min \\
\hline Setup reduction factor & 0.5 \\
\hline Transfer times & Uniformly distributed (1 5 min) for CL \\
\hline Job sequencing rule & Uniformly distributed (1 15 min) for FL \\
\hline
\end{tabular}

The first experiment studies the effect of part type routing on comparison results using three surrogate sets of routings in both layouts. In the first routing set, every part type requires from 2 to 6 operations. The second set is obtained by altering the first while conserving the 
number of manufacturing operations per part type. The third set of routings corresponds to the first with a lowered number of manufacturing operations per part type. All sets present full cell independence.

In the second experiment, a version of the CL model without general cell waiting queues is compared to the basic CL and FL models. Using this modified version, every job is directly routed to the first machine of its routing even if this machine is not idle.

The third and final experiment aims at investigating the effect of job overlapping on comparison results. Hence, FL-CL comparison is undertaken in two contexts in which overlapping is first allowed and then blocked.

\subsection{Simulation setup}

The independent replications method is adopted for statistics collection. So, every simulation run has 15 replications. Each replication is 1.000.000 minutes long. Observations are collected for two performance measures: throughput time and mean flow time. The first measure is essentially used for warm up period detection. Nevertheless, FL-CL comparison is based on the second measure. Analysis of the simulation output using the Yucesan initialization bias in simulation output detection method shows that the steady state is reached when the simulation length is 200.000 minutes [17]. As for the second performance measure, $95 \%$ confidence interval $(C I)$ is considered. Therefore, in this study, a difference between two MFT measures is considered "significant" only when there is no overlap between their respective $95 \%$ confidence intervals. The confidence interval can be calculated using Eq. 1 [18]:

$$
C I=m \pm t \frac{\sigma}{\sqrt{n}}
$$

where $m$ is the average MFT over the $n$ the number of replications, $t$ is the factor obtained from standard student table required for the risk $\alpha, \sigma$ is the MFT standard deviation. In this study the risk $\alpha$ is equal to $0.05(1-0.95)$ and $n$ is equal to 15 . The corresponding factor $t$ is so equal to 2.14 .

\subsection{Results}

Simulation results for the first experiment, depicted in Table II, clearly establish the influence of part type routings on the simulation results. Indeed, with the first routing the CL's MFT is $9.4 \%$ lower than the FL's MFT. The superiority between layouts is though annulled by the second routing since the two layouts generate significantly equivalent MFT. On the other hand, the third routing inverts the superiority between CL and FL and the FL generate an MFT $22 \%$ lower than the CL's MFT. Therefore, the superiority of a layout over the second could be reversed when the part routing set is altered.

For the second experiment, results show that the elimination of the cell general queue decreases the gap between the performances of the two layouts. Indeed, when the general queue is used in the CL, this layout outperforms the FL and the difference between the two layouts MFT reaches 134 minutes whereas the same difference is not significant when the general queue used in the CL (see Table II).

Finally, results of the last experiment clearly reveal, that job overlapping influences the comparison results. Indeed the FL generates an MFT 5.75 \% lower than the CL's MFT if job overlapping is allowed. On the opposite, CL outperforms the FL in case of job overlapping not allowed (see Table II). 
Table II: Simulation results (MFT confidence interval).

EXPERIMENT I: part type routing effect

\begin{tabular}{|c|c|c|c|c|c|c|}
\hline \multirow{2}{*}{ Routing } & \multicolumn{3}{|c|}{$M F T_{F L}$} & \multicolumn{3}{|c|}{$M F T_{C L}$} \\
\hline & $m$ & $\sigma$ & $C I$ & $m$ & $\sigma$ & $C I$ \\
\hline First set & 1558 & 14 & [1550-1566] & 1424 & 13 & [1417-1431] \\
\hline Second set & 1534 & 13 & [1527-1541] & 1537 & 21 & [1525-1549] \\
\hline Third set & 1162 & 7 & [1158-1166] & 1417 & 18 & [1407-1427] \\
\hline
\end{tabular}

EXPERIMENT II: cellular queuing strategy

\begin{tabular}{|c|c|c|c|c|c|c|}
\hline \multirow{2}{*}{ Cell } & \multicolumn{3}{|c|}{$M F T_{F L}$} & \multicolumn{3}{|c|}{$M F T_{C L}$} \\
\hline & $m$ & $\sigma$ & $C I$ & $m$ & $\sigma$ & $C I$ \\
\hline With general queue & & & & 1424 & 13 & {$[1417-1431]$} \\
\hline & 1558 & 14 & [1550-1566] & & & \\
\hline Without general queue & & & & 1546 & 12 & [1540-1552] \\
\hline
\end{tabular}

EXPERIMENT III: overlapping effect

\begin{tabular}{|c|c|c|c|c|c|c|}
\hline \multirow{2}{*}{ Overlapping } & \multicolumn{3}{|c|}{$M F T_{F L}$} & \multicolumn{3}{|c|}{$M F T_{C L}$} \\
\hline & $m$ & $\sigma$ & $C I$ & $m$ & $\sigma$ & $C I$ \\
\hline Not allowed & 1558 & 14 & [1550-1566] & 1424 & 13 & [1417-1431] \\
\hline Allowed & 3891 & 25 & [3877-3905] & 4115 & 20 & [4104-4126] \\
\hline
\end{tabular}

Notes: $m$ - average MFT (min), $\sigma-M F T$ standard deviation (min), CI - Confidence Interval (min).

\section{CONCLUSION AND FUTURE WORK}

This paper focuses on the functional and cellular layout comparison. It mainly presents comprehensive simulation models for both layouts. These models are then used to illustrate how some parameters, often overlooked in FL-CL comparison studies, can influence comparison between layouts results. Indeed, the simulation results proved that part type routings, cell queuing strategy and job overlapping strategy affect the relative superiority of the two layouts.

Undergoing work focuses on investigation of other parameters effects on the system performances. Besides, developed layout models in this study are being used in establishing an objective simulation-based comparison methodology. Such a methodology is based on the Taguchi method for robust experimental design.

\section{REFERENCES}

[1] Morris, J. S.; Tersine, R. J. (1990). A simulation analysis of factors influencing the attractiveness of group technology cellular layouts, Management Science, Vol. 36, No. 12, 1567-1578

[2] Morris, J. S.; Tersine, R. J. (1994). A simulation comparison of process and cellular layouts in a dual resource constrained environment, Computers and Industrial Engineering, Vol. 26, No. 4, 733-741

[3] Jensen, J. B.; Malhotra, M. K.; Philipoom, P. R. (1996). Machine dedication and process flexibility in a group technology environment, Journal of Operations Management, Vol. 14, No. 1, 19-39

[4] Shafer, S. M.; Charnes, J. M. (1992). Cellular versus functional layouts under a variety of shop operating conditions, Decision Sciences, Vol. 24, No. 3, 665-681 
[5] Pitchuka, L. N.; Adil, G. K.; Ananthakumar, U. (2006). Effect of the conversion of the functional layout to a cellular layout on the queue time performance: some new insights, International Journal of Advanced Manufacturing Technology, Vol. 31, No. 5/6, 594-601

[6] Suresh, N. C.; Meredith, J. R. (1994). Coping with the loss of pooling synergy in cellular manufacturing systems, Management Science, Vol. 40, No. 4, 466-483

[7] Shafer, S. M.; Charnes, J. M. (1995). A simulation analysis of factors influencing loading practices in cellular manufacturing, International Journal of Production Research, Vol. 33, No. $1,279-290$

[8] Farrington, P. A.; Nazametz, J. W. (1998). Evaluation of the performance domain of cellular and functional layouts, Computers and Industrial Engineering, Vol. 34, No. 1, 91-101

[9] Faizul, H.; Douglas, A. H.; Zubair, M. M. (2001). A simulation analysis of factors influencing the flow time and through-put performance of functional and cellular layouts, Integrated Manufacturing Systems, Vol. 12, No. 4, 285-295

[10] Li, J. (2003). Improving the performance of job shop manufacturing with demand-pull production control by reducing set-up/processing time variability, International journal of Production Economics, Vol. 84, No. 3, 255-270

[11] Shambu, G.; Suresh, N. C.; Pegels, C. C. (1996). Performance evaluation of cellular manufacturing systems: a taxonomy and review of research, International Journal of Operations \& Production Management, Vol. 16, No. 8, 81-103

[12] Chtourou, H.; Jerbi, A.; Maalej, A. Y. (2008). The cellular manufacturing paradox: a critical review of simulation, Journal of Manufacturing Technology Management, Vol. 19, No. 5, 591606

[13] Agarwal, A.; Sarkis, J. (1998). A review and analysis of comparative performance studies on functional and cellular manufacturing layouts, Computers and Industrial Engineering, Vol. 34, No. 1, 77-89

[14] Arena Standard User's Guide (2002). Doc ID ARENAS-UM001C-EN-P, Rockwell Software Inc.

[15] Kelton, W. D.; Sadowski, R. P.; Sadowski, D. A. (2002). Simulation with Arena, McGraw-Hill, New York

[16] Jerbi, A.; Chtourou, H.; Maalej, A. Y. (2006). Functional VS Cellular layout: using simulation as a comparison tool, The Third International Conference on Advances in Mechanical Engineering and Mechanics, Hammamet, Tunisia

[17] Yucesan, E. (1993). Randomization tests for initialization bias in simulation output, Naval Research Logistics Quarterly, Vol. 40, 643-663

[18] Kiefer, J. (1977). Conditional Confidence Statements and Confidence Estimators (with discussion), Journal of the American Statistical Association, Vol. 72, 789-827 